\title{
Changes in Global and Regional Mechanics Due to Atrial Fibrillation: Insights from a Coupled Finite-Element and Circulation Model
}

\author{
Christian B. Moyer, ${ }^{1}$ Patrick T. Norton, ${ }^{3}$ John D. Ferguson, ${ }^{2}$ and Jeffrey W. Holmes ${ }^{1,2,4}$ \\ ${ }^{1}$ Department of Biomedical Engineering, University of Virginia, P.O Box 800759, Charlottesville, VA 22908, USA; ${ }^{2}$ Department \\ of Medicine, University of Virginia, Charlottesville, VA, USA; ${ }^{3}$ Department of Radiology, University of Virginia, \\ Charlottesville, VA, USA; and ${ }^{4}$ Robert M. Berne Cardiovascular Research Center, University of Virginia, Charlottesville, \\ VA, USA
}

(Received 17 September 2014; accepted 14 January 2015; published online 29 January 2015)

Associate Editor Estefanía Peña oversaw the review of this article.

\begin{abstract}
Atrial fibrillation (AF) is a rhythm disorder with rapidly increasing prevalence due to the aging of the population. AF triggers structural remodeling and a gradual loss of function; however, the relative contributions of specific features of AF-induced remodeling to changes in atrial mechanical function are unclear. We constructed and validated a finite-element model (FEM) of the normal human left atrium using anatomic information from cardiac magnetic resonance imaging, material properties and fiber orientations from published studies, and an iterative algorithm to estimate unloaded geometry. We coupled the FEM to a circuit model to capture hemodynamic interactions between the atrium, pulmonary circulation, and left ventricle. The normal model reproduced measured volumes within 1 $\mathrm{SD}$, as well as most metrics of regional mechanics. Using this validated human model as a starting point, we explored the impact of individual features of atrial remodeling on atrial mechanics and found that a combination of dilation, increased pressure, and fibrosis can explain most of the observed changes in mechanics in patients with paroxysmal AF. However, only impaired ventricular relaxation could reproduce the increased reliance on active emptying we observed in these patients. The resulting model provides new insight into the mechanics of $\mathrm{AF}$ and a platform for exploring future therapies.
\end{abstract}

Keywords-Cardiac mechanics, Cardiac function, Atrial function, Atrial geometry, Atrial remodeling, Magnetic resonance imaging.

Address correspondence to Jeffrey W. Holmes, Department of Biomedical Engineering, University of Virginia, P.O Box 800759, Charlottesville, VA 22908, USA. Electronic mail: holmes@ virginia.edu

\section{INTRODUCTION}

Atrial fibrillation $(\mathrm{AF})$ is a rhythm disorder in which the normal process of coordinated atrial electrical activation and mechanical contraction is disrupted, resulting in a dramatic drop in atrial mechanical function and stasis of blood that greatly increases the risk of stroke. ${ }^{37}$ The prevalence of AF increases with age, from $0.1 \%$ in people younger than 55 to $9.0 \%$ in people over $80 .{ }^{12} \mathrm{At}$ the onset, AF is typically episodic, but the episodes trigger remodeling that increases the likelihood and severity of future episodes: the atrium dilates ${ }^{33}$ and becomes more spherical, ${ }^{11}$ pressures increase ${ }^{35}$ the wall becomes fibrotic, ${ }^{30}$ and conduction slows. ${ }^{29}$

Atrial contraction (often referred to as "active emptying") contributes approximately $30 \%$ of left ventricular stroke volume in healthy adults $(20$ 29 years old). ${ }^{4}$ Therefore, episodes of AF significantly impair heart function, doubling atrial pressures and lowering cardiac output. ${ }^{5}$ Importantly, remodeling triggered by AF decreases atrial function even in the periods between episodes. ${ }^{3,28}$ While some of the features of atrial remodeling have been identified as risk factors for future AF episodes, their relative contributions to AF-induced changes in atrial mechanical function are unclear. In order to better understand the role of these factors, we constructed and validated a finite-element model of the human left atrium and used it to explore the impact of changes in atrial size, shape, pressures, fibrosis, and conduction velocity on atrial mechanics.

Atrial fibrillation in the absence of other cardiovascular disease ("lone" AF) represents less than 5\% of all diagnosed cases; ${ }^{10}$ more typically, AF develops alongside conditions such as hypertension, congestive 
heart failure, and mitral regurgitation, which alter ventricular function and systemic hemodynamics. Therefore, understanding changes in atrial function in context requires understanding how accompanying systemic changes impact the atrium. Accordingly, we coupled our finite-element model of the left atrium to a circulation model to account for changes in filling and emptying due to both atrial and systemic factors. To our knowledge, the coupled FEM-circulation model presented here represents the first model of human left atrial mechanics validated in both normal subjects and patients with a history of atrial fibrillation. This model provided two important insights that we hope will improve understanding of the mechanics of AF and guide development of future therapies: (1) dilation and increased pressures in the atrium are likely the most important atrial factors in reducing atrial function in patients with a history of AF; and (2) the increased reliance on active emptying in patients with paroxysmal AF appears to be due to impaired ventricular relaxation rather than changes in the atrium itself.

\section{METHODS}

\section{Validation Data}

Two sources of data were used in construction and validation of the model described below. Average geometries, volumes, and regional wall motion throughout the cardiac cycle were obtained from magnetic resonance imaging (MRI) studies of 10 healthy volunteers and 31 patients with paroxysmal AF who were in sinus rhythm at the time of a routine MRI prior to planned radiofrequency $(\mathrm{RF})$ catheter ablation. These data and methods for fitting and analyzing endocardial surface motion were reported previously. ${ }^{27,28}$ Additionally, in 23 paroxysmal AF patients and in 6 patients with non-atrial arrhythmias (Wolff-Parkinson-White syndrome), we also obtained intra-operative pressure-volume data using a Millar conductance catheter (SPC-550-5, Millar Instruments, Houston, TX) immediately prior to RF ablation. ${ }^{27}$ Conductance catheter volumes were calibrated against volumes from a pre-procedure MRI performed on a separate day; therefore, where volumes from the two patient groups differed, we gave preference to directly measured MRI volumes when validating the model. All studies were approved by the University of Virginia Institutional Review Board and performed with informed consent.

\section{Normal Atrium FEM: Geometry and Fiber Structure}

We constructed a finite-element model (FEM) of the normal human left atrium using the finite-element software FEBio v1.5 (Musculoskeletal Research Lab, University of Utah, Salt Lake City, $\mathrm{Utah}^{25}$ ). We specified the geometry based on an average endocardial surface constructed from fits to contoured MR images of ten healthy subjects. ${ }^{28}$ We began with a spherical mesh of constant wall thickness with 30,000 hexahedral elements arranged in two transmural layers in PreView (MRL, University of Utah). In MATLAB, we converted the position of all nodes into spherical coordinates and projected the endocardial nodes along their radial dimension onto the MRI-derived surface. We assumed a constant wall thickness of $3.0 \mathrm{~mm}$ and positioned midwall and epicardial nodes at equal distances from corresponding endocardial nodes along the local surface normal. The resulting atrial mesh is displayed in Fig. 1 with the epicardial surface visible in red. Preliminary simulations with three different mesh densities showed that atrial volumes changed less than $5 \%$ between meshes of 19,200 and 30,000 elements.

Published anatomic ${ }^{17}$ and histologic ${ }^{38}$ studies suggest that muscle fiber orientations in the left atrium do not vary smoothly from region to region or across the thickness of the wall as in the left ventricle. Furthermore, these studies show that fibers are oriented close to the circumferential or longitudinal direction in many regions of the atrium. We therefore subdivided the atrium into one posterior (bounded by the pulmonary veins) and four equatorial regions (lateral, inferior, septal, and superior, each running between the pulmonary vein plane and mitral annulus), ${ }^{28}$ then prescribed circumferential or longitudinal fiber orientations separately in endocardial and epicardial elements in two halves of each region (Table 1).

The pulmonary veins and mitral valve annulus provide important physical constraints on the deformation of the left atrium. We used the position of the four pulmonary vein ostia and four annular points along the mitral valve in the MRI-derived average healthy geometry ${ }^{28}$ to specify these structures in the model. For the pulmonary veins, we projected each ostium onto the epicardial side of the mesh at the nearest epicardial node and created hollow cylinders $1.5 \mathrm{~mm}$ thick, with diameters ranging from 4 to $6 \mathrm{~mm},{ }^{18,34}$ composed of 200 hexahedral elements and oriented along the local surface normal. For the mitral annulus, we created a thin ring composed of 80 elements, oriented in the plane of the mitral valve landmarks and attached to the epicardial side of the atrium. As the pulmonary veins and the mitral annulus were included in the model for the sole purpose of enforcing displacement boundary conditions (see below), we modeled them as rigid bodies.

\section{Normal Atrium FEM: Material Properties}

DiMartino and colleagues recently performed biaxial testing of porcine left atria and reported fitted 
(a)

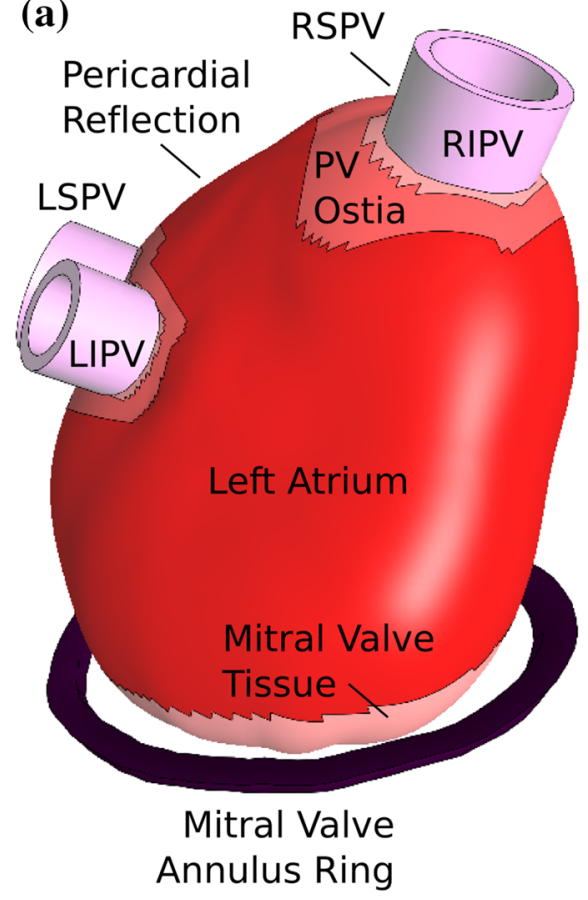

(b)

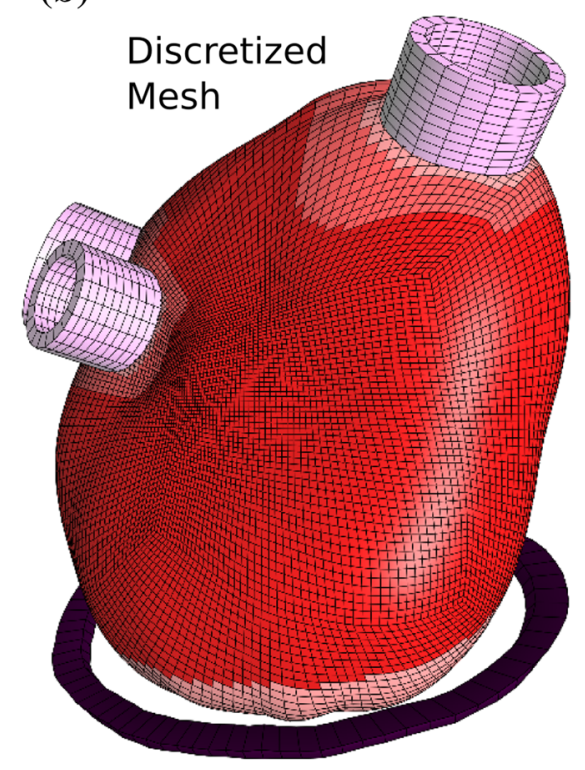

(c)
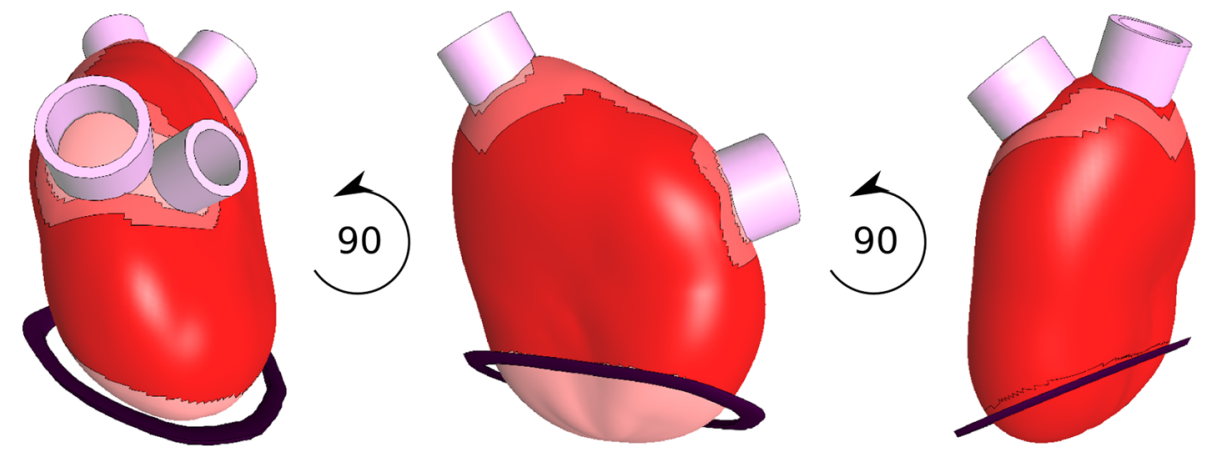

FIGURE 1. Geometry of normal left atrial model at a cavity pressure of $5 \mathrm{mmHg}$. (a) The four pulmonary veins are labeled as right superior (RSPV), right inferior (RIPV), left superior (LSPV), and left inferior (LIPV). (b) Finite-element mesh used for computations $\left(30,000\right.$ elements). (c) Additional views of the model rotated in $90^{\circ}$ steps.

coefficients of a transversely isotropic strain energy function. ${ }^{2}$ We selected a strain-energy function available in FEBio with identical isotropic terms, allowing us to use Bellini's $C_{1}$ and $C_{2}$ values directly:

$$
\begin{array}{r}
W=C_{1}\left(I_{1}-3\right)+C_{2}\left(I_{2}-3\right)+F_{2}(\lambda)+(K / 2) \ln (J)^{2}, \\
d F_{2} / d \lambda=C_{3} / \lambda *\left(\exp \left(C_{4}(\lambda-1)\right)-1\right)
\end{array}
$$

where $W$ is the strain energy, $C$ is the right CauchyGreen strain tensor, $I_{1}$ and $I_{2}$ are the first and second invariants of $C, \lambda$ is the stretch ratio in the fiber direction, $J$ is the determinant of $C$, and $K$ is a bulk modulus set to a high value to enforce incompressibility. We then used Bellini's strain energy function to generate stress-strain curves for a range of biaxial test protocols, refitted those data to obtain initial estimates of $C_{3}$ and $C_{4}$, and manually adjusted these parameters to match our measured volume changes. The final material parameters for our normal atrial model were: $C_{1}=1.65 \mathrm{kPa}, C_{2}=0 \mathrm{kPa}, C_{3}=0.015 \mathrm{kPa}, \quad$ and $C_{4}=13.37$; the bulk modulus was set to $K=500 \mathrm{kPa}$, the maximum possible value that did not cause element locking. To reduce stress concentrations near the (rigid) pulmonary veins and mitral annulus, we stiffened neighboring elements by modifying the atrial material constants $\left(C_{1}=16.5 \mathrm{kPa}, C_{2}=1, C_{3}=0.150 \mathrm{kPa}\right)$. We simulated active contraction by adding a lengthdependent active stress along the local fiber direction, following Guccione et al.: ${ }^{14}$

$$
T_{\text {active }}=T_{\max } *\left[\left(\mathrm{Ca}_{0}^{2}\right) /\left(\mathrm{Ca}_{0}^{2}+E \mathrm{Ca}_{50}^{2}\right)\right] * C(t)
$$

where $T_{\max }$ is the maximum isometric stress, $\mathrm{Ca}_{0}$ is the peak intracellular calcium concentration, $E \mathrm{Ca}_{50}$ is the 
TABLE 1. Atrial fiber angles by region.

\begin{tabular}{|c|c|c|c|c|c|}
\hline Region & Subregion & Endo/Epi & Ho 2002 & Zhao 2013 & Model \\
\hline \multirow[t]{4}{*}{ Posterior } & Superior & Endo & Long & Circ & Long \\
\hline & & Epi & Long & Long & Long \\
\hline & Inferior & Endo & & Circ & Circ \\
\hline & & Epi & Long & Long & Long \\
\hline \multirow[t]{4}{*}{ Inferior } & Posterior & Endo & & Long & Long \\
\hline & & Epi & Long & Circ & Long \\
\hline & Annular & Endo & & Long & Long \\
\hline & & Epi & Circ & Circ & Circ \\
\hline \multirow[t]{4}{*}{ Septal } & Posterior & Endo & & Long & Long \\
\hline & & Epi & & Long & Long \\
\hline & Annular & Endo & & Long & Long \\
\hline & & Epi & & Long & Long \\
\hline \multirow[t]{4}{*}{ Superior } & Posterior & Endo & Long & Long & Long \\
\hline & & Epi & Circ & Circ & Circ \\
\hline & Annular & Endo & Long & Long & Long \\
\hline & & Epi & Circ & Circ & Circ \\
\hline \multirow[t]{4}{*}{ Lateral } & Posterior & Endo & Circ & Circ & Circ \\
\hline & & Epi & Circ & Circ & Circ \\
\hline & Annular & Endo & Circ & Circ & Circ \\
\hline & & Epi & Circ & Circ & Circ \\
\hline
\end{tabular}

The left atrium was divided into five regions, each with two subregions with endocardial and epicardial sides. Fiber angles were approximated as either circumferential (Circ) or longitudinal (Long) from two reference studies, then reconciled to form the atrial material model fibers.

length-dependent calcium sensitivity, and $C(t)$ is a scaling variable that depends on both time after onset of contraction and sarcomere length. All parameters were identical to those employed by Guccione for the left ventricle except $T_{\max }=10 \mathrm{kPa}$, chosen to provide a reasonable match to the average volumes ejected during active atrial contraction in our healthy volunteers.

\section{Normal Atrium FEM: Loading and Boundary Conditions}

The FEM was loaded using prescribed pressure vs. time curves (Fig. 2) measured immediately prior to AF ablation in 23 patients with paroxysmal $\mathrm{AF}^{27}$ and scaled to adjust the maximum and minimum pressures during filling, passive emptying, and active emptying by coupling the FEM to a circuit model of the upstream and downstream circulation as described below. The attachment point of the pulmonary veins (PVs) to the left atrium only moves a few millimeters during a normal cardiac cycle ${ }^{28}$ this physical constraint was represented in the model by prescribing zero displacement of the pulmonary veins for all modeling scenarios. By contrast, the mitral valve (MV) moves like a piston during the cardiac cycle, with large downward excursions during atrial filling (ventricular systole) driven by contraction of the ventricle and papillary muscles, followed by recoil during atrial emptying (ventricular diastole). ${ }^{28}$ To recreate this motion in the normal atrium model, we prescribed the MV displacement we measured along the PV-MV axis (the $z$ axis of our model coordinate system) using MRI in healthy volunteers, ${ }^{28}$ calculated the reaction forces required to achieve the prescribed displacement, and applied this force boundary condition in all future simulations. Thus, model atria with altered material properties, geometry, etc. experienced identical downward forces due to LV contraction, but not identical MV displacements.

Finally, we accounted for physical contact between the thin-walled atrium and surrounding structures. The effect of this contact is apparent in the shape of left atrium; for example, the concave indentation in the center of the superior wall persists even when the thinwalled atrium is pressurized, due to contact from the aortic root (Fig. 3). We simulated this contact using an inward-facing pressure applied to every epicardial element in this region that contained a negative curvature node. We modeled right atrial pressure as an inward-facing pressure on the epicardial face of the septal wall, scaled to $30 \%$ of the left atrial pressure based on literature values ${ }^{15}$ and measured septal wall motion. ${ }^{28}$ Finally, we applied an external pressure to the posterior wall, where the atrium touches the chest wall, and scaled the magnitude of the pressure to allow a small amount of expansion consistent with our MRI measurements.

\section{Estimating Unloaded Geometry in the FEM}

The finite-element method uses a stress-free configuration as the reference state, yet stress-free states 


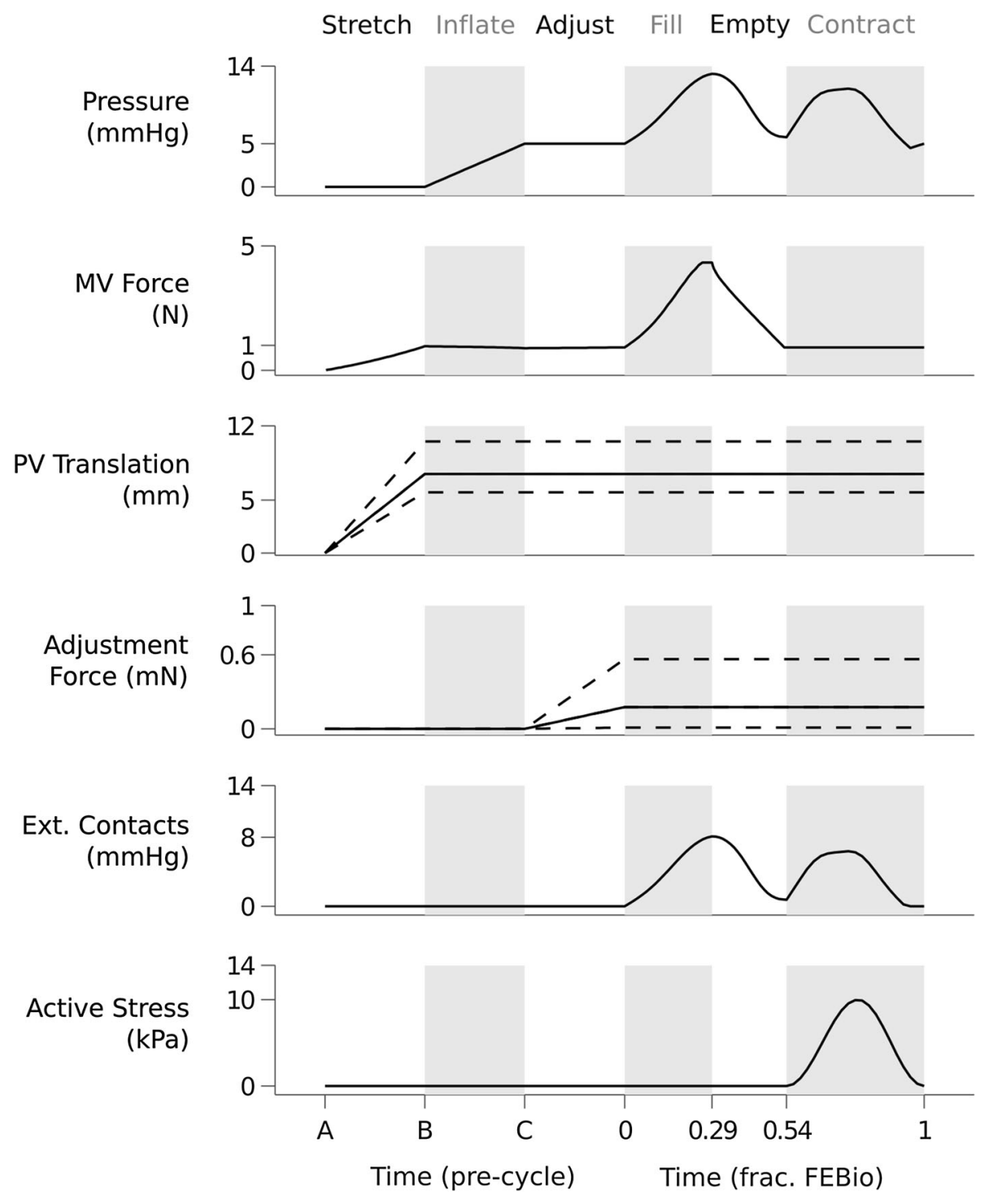

FIGURE 2. Load curves applied to the finite-element model; see text for explanation of first 3 loading steps. Atrial chamber pressure, force applied to the mitral valve (MV) annulus, displacement boundary conditions applied to the pulmonary veins (PV), shape adjustments, external contact pressure applied to the aortic root (scaled versions applied to the septal and posterior chest wall), and active stress are all shown. Dotted lines show the range across nodes where appropriate.

rarely exist inside the body. The atrial geometry constructed here was based on MRI images taken at the time of minimum atrial pressure (the start of passive filling), but nevertheless reflected a pressurized, loaded state. Several techniques have been developed to solve the so-called inverse problem of estimating an unknown, unloaded geometry from a known, loaded geometry; ${ }^{13,32}$ we employed an inverse displacement method most similar to that described by Raghavan et al..$^{32}$ First, we guessed an unloaded shape by scaling the mesh radius of every endocardial node to $80 \%$ of its original value. Next, we displaced the nodes nearest the MV onto the fixed MV annulus ring and the nodes nearest the pulmonary veins to meet the model PV cylinders ("stretch" phase in Fig. 2), and inflated the mesh to an internal pressure of $5 \mathrm{mmHg}$, keeping both the annulus and veins fixed ("inflate" phase). We used $5 \mathrm{mmHg}$ as the minimum atrial pressure during a normal cardiac cycle, based on average atrial pressures in our experiments ${ }^{27}$ and literature reports. ${ }^{21,36} \mathrm{We}$ calculated the difference between the inflated mesh and the reference surface, then updated the estimated unloaded geometry by subtracting $70 \%$ of the difference. We iterated three times until the solution reached equilibrium and treated the final estimate as the unloaded, stress-free state for future simulations. Because the final shape was known and potentially important, in the final simulation we applied small adjustment 
(a)
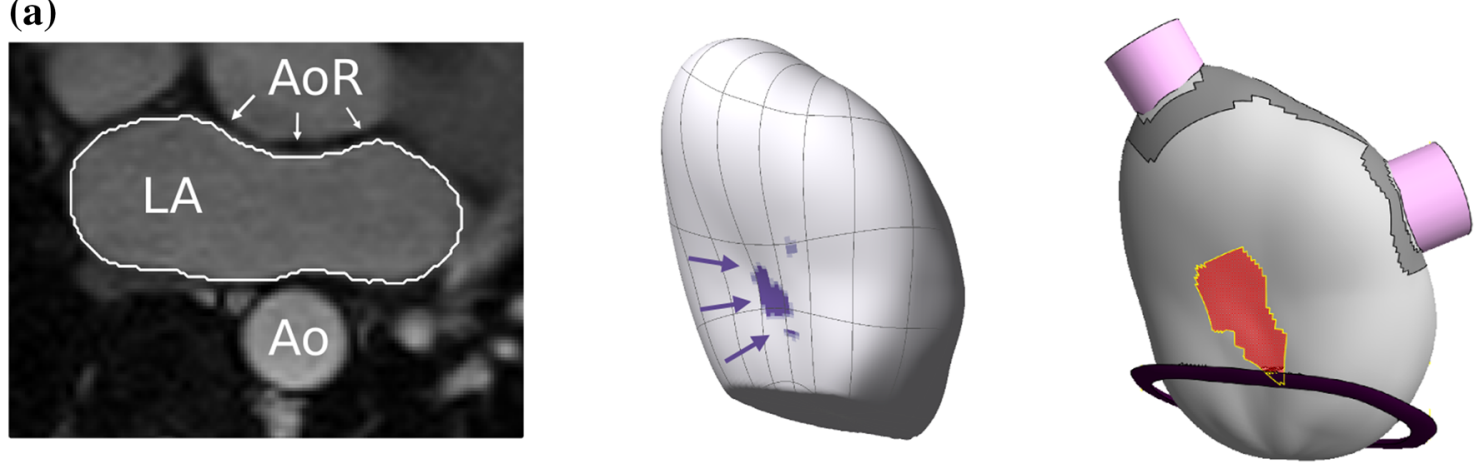

(b)
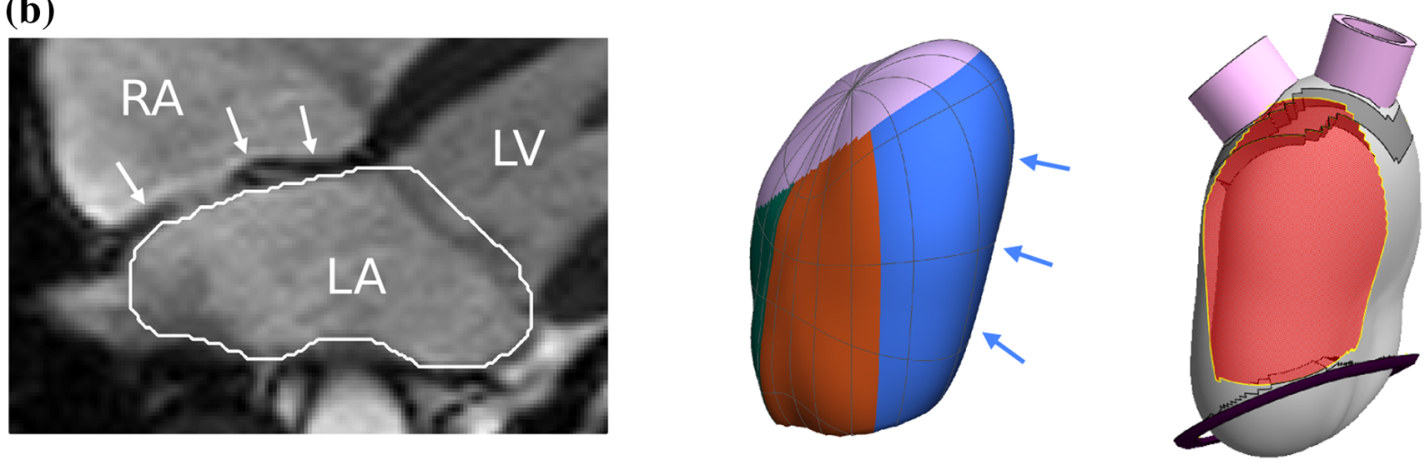

(c)
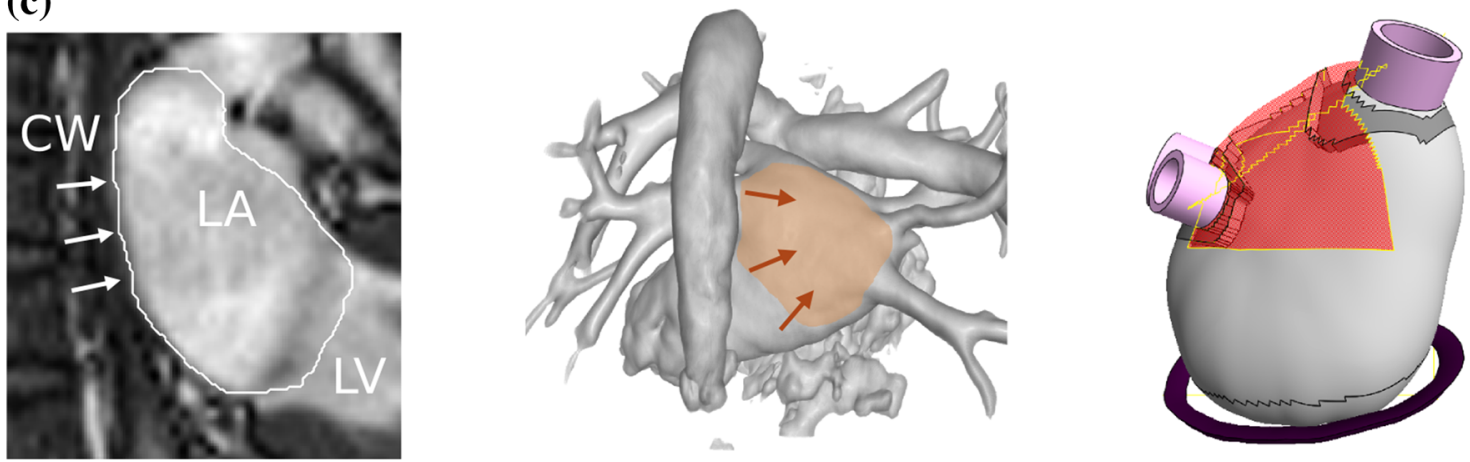

FIGURE 3. Simulation of contact with surrounding structures by applying external pressures. (a) Concave region of the aortic root (AoR), visible on axial MRI and in surface curvature. (b) Contact with the right atrium (RA) across the atrial septum, visible on 4-chamber MRI and in regional segmentation of surface. (c) Posterior chest wall (CW), visible on 2-chamber MRI and 3-D magnetic resonance angiogram. LA - left atrium; LV - left ventricle.

forces ("adjust" phase) to correct for remaining errors in shape following inflation.

\section{Circulation Model}

During the cardiac cycle, the atrium expands and contracts under varying chamber pressure. The range of this variation is governed both by the properties of the atrium itself and by interaction with the pulmonary circulation (upstream) and left ventricle (downstream). We therefore coupled our atrial FE model to a hydraulic circuit model of the pulmonary vasculature, mitral valve, and left ventricle (Fig. 4) proposed by Alexander et al. ${ }^{1}$ Atrial filling was driven by an upstream pressure in the pulmonary vasculature $\left(P_{\mathrm{V}}=18 \mathrm{mmHg}\right.$ during ventricular systole, $13 \mathrm{mmHg}$ during ventricular diastole) and resistance to flow in the pulmonary veins modeled with a single resistor $\left(R_{\mathrm{PV}}=0.075 \mathrm{mmHg} * \mathrm{~s} / \mathrm{mL}\right)$. We represented the mitral valve as a resistor and inductor in series with values $R_{\mathrm{MV}}=0.018 \mathrm{mmHg}^{*} \mathrm{~s} / \mathrm{mL}$, $L_{\mathrm{MV}}=0.0007 \mathrm{mmHg} * \mathrm{~s}^{2} / \mathrm{mL}$. The passive properties of the ventricle were modeled using an exponential function:

$$
P_{\mathrm{LV}, \text { passive }}=B_{1} *\left(\exp \left(B_{2} *\left(V_{\mathrm{LV}}-V_{\mathrm{LV}, 0}\right)\right)-1\right),
$$




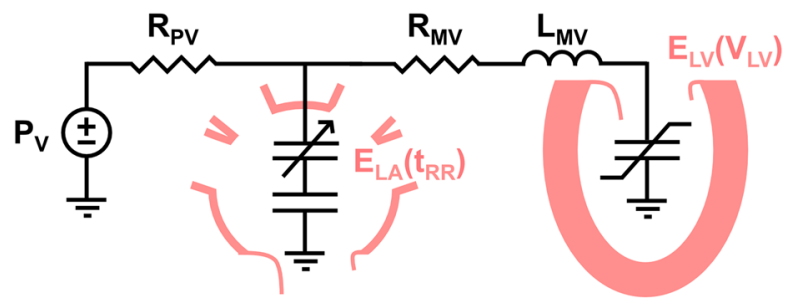

FIGURE 4. Hydraulic circuit model of blood flow. During atrial filling, mitral valve is closed and LA fills from pulmonary veins with pulmonary venous pressure $P_{v}$ and pulmonary vein resistance $R_{\text {Py. }}$. Passive atrial properties are represented by an exponential $\boldsymbol{P}-\boldsymbol{V}$ curve generated in the FEM. During passive emptying, mitral valve opens and blood flows through the mitral valve resistor $R_{M V}$ and inductor $L_{M V}$, filling the left ventricle (LV) along its exponential end-diastolic pressurevolume relationship (EDPVR). During active emptying, the atrium contracts with active elastance $E_{L A}$ until it reaches the peak systolic pressure-volume curve predicted by the FEM.

with coefficients $B_{1}=3.36 \mathrm{mmHg}$ and $B_{2}=$ $0.011 \mathrm{~mL}^{-1}$ and an unloaded ventricular volume of $V_{\mathrm{LV}, 0}=8 \mathrm{~mL}$, chosen to achieve an end-diastolic volume of $120 \mathrm{~mL}$ at $8 \mathrm{mmHg}$ internal pressure and a minimum volume of $50 \mathrm{~mL}$ at a minimum pressure of $2.5 \mathrm{mmHg}$. Active LV contraction was modeled using a time-varying elastance model:

$$
P_{\mathrm{LV}, \text { active }}=E_{\mathrm{LV}}(t) *\left(V_{\mathrm{LV}}-V_{\mathrm{LV}, 0}\right),
$$

with $E_{\mathrm{LV}}(t)$ ranging from 0.1 to $2.4 \mathrm{mmHg} / \mathrm{mL}$.

Atrial passive and active behavior were modeled with similar exponential and time-varying elastance curves, but parameters were determined by the coupled FEM as explained in the next section. The simulated length of each phase of the atrial cardiac cycle was based on our measured average human atrial $P-V$ data: ${ }^{27}$ passive filling for $0.25 \mathrm{~s}(29 \% R-R)$, passive emptying for $0.212 \mathrm{~s}(25 \%)$, and active contraction for $0.393 \mathrm{~s}(46 \%)$, at a heart rate of 70 beats per minute. We did not allow retrograde flow across the mitral valve but did allow reverse flow in the pulmonary veins. We solved the set of ordinary differential equations for time-varying pressures, volumes, and flows in each chamber using Euler's method.

\section{Coupling the Finite-Element and Circulation Models}

In vivo, the atrium is fully coupled to the circulation: any change in atrial properties - such as an increase in passive stiffness - could alter passive filling, active force generation, and pressures in the atrium. As we simulated changes in atrial size, shape, and stiffness in the FEM, we therefore updated the circuit model to reflect the resulting changes in passive and active properties of the atrium, and in turn used the circuit model to update the pressure loading curves specified in the FEM. For each new version of the FEM, we simulated passive atrial inflation and fitted the predicted passive pressure-volume curve to the threeparameter exponential function used to represent passive atrial properties in the circuit model. Then, we simulated active contraction of the atrium until the model reached peak active stress, fixed the stress state in the model, and inflated the contracted chamber; we fit the resulting systolic atrial $P-V$ curve to a straight line, the slope of which provided the maximal elastance $\left(E_{\mathrm{LA}, \max }\right)$ and x-intercept $\left(V_{\mathrm{LA}, 0}\right)$ values used to simulate atrial contraction in the circuit model. Finally, we ran the adapted circuit model until it reached equilibrium to determine the minimum and maximum pressures for each phase of the atrial cycle. We then scaled the pressure loading curves (Fig. 2) for the FEM to match these new pressure values, and completed a final FE simulation of the full atrial cycle.

\section{Simulating Features of Atrial Fibrillation in the Coupled Model}

Patients experience many physiological changes during the progression of AF. We investigated five factors that are known to change in patients with AF and might be expected to alter mechanics: (1) size, (2) shape, (3) pressure, (4) fibrosis, and (5) conduction. We simulated changes in each of these factors consistent with literature reports from AF patients and assessed their relative impact on mechanical function in the coupled model. We simulated an increase in size (but not shape) consistent with a history of paroxysmal AF by increasing the radius of every endocardial node by a constant factor to match the average volume we measured at the beginning of passive filling in these patients. ${ }^{28}$ We simulated a change in shape typical of paroxysmal AF by scaling down the average geometry we measured in AF patients ${ }^{28}$ to match normal atrial volumes. We modeled increases in atrial pressure by altering the pressure load curve in the baseline model; based on published studies ${ }^{21,35}$ and our own measurements, ${ }^{27}$ we selected $10 \mathrm{mmHg}$ as a typical minimum pressure in paroxysmal AF patients and scaled the upstream pulmonary pressure and downstream ventricular pressure proportionally to maintain reasonable flow rates. We estimated the change in material properties expected due to fibrosis from the relationship between collagen content and material properties measured by Fomovsky et al. in healing rat infarcts $^{9}$ and literature estimates of collagen content in AF atria of approximately $25 \%,{ }^{30}$ resulting in a choice of $C_{2}=1.0 \mathrm{kPa}\left(C_{1}, C_{3}, C_{4}, K\right.$ unchanged $)$ as atrial material parameters for the fibrosis simulation. Finally, we simulated slowed conduction associated with AF by staggering the activation of different regions in the finite-element model, which also led to prolonged 
atrial systole with a diminished $E_{\max }$ in the circuit model; based on measurements in patients with paroxysmal AF, we reduced simulated conduction velocity from $90 \mathrm{~cm} / \mathrm{s}$ (time delay of $33 \mathrm{~ms}$ from earliest to latest activation) to $69 \mathrm{~cm} / \mathrm{s}(43 \mathrm{~ms}){ }^{26}$

\section{RESULTS}

\section{Normal Atrium: Unloaded Geometry}

We iteratively guessed an unloaded geometry for the normal left atrium, inflated it to a pressure of $5 \mathrm{mmHg}$, compared the pressurized geometry to the average geometry we measured at the start of passive atrial filling in ten healthy volunteers, ${ }^{28}$ and corrected the unloaded geometry based on the difference (see Methods). After three iterations, the final inflated geometry differed by $0.3 \mathrm{~mL}$ in volume $(1 \%)$ and had a radius RMSE of $0.8 \mathrm{~mm}(4 \%)$; additional iterations did not improve the RMSE but tended to introduce local variations in the surface radii. Final shape adjustments of $0.2 \pm 0.4 \mathrm{mN}$ lowered the RMSE to $0.1 \mathrm{~mm}(<1 \%)$.

\section{Normal Atrium: Validation Against Measured Volumes and Hemodynamics}

The normal atrial model filled to a maximum volume of $66 \mathrm{~mL}$, emptied passively to $39 \mathrm{~mL}$ in diastasis, and contracted to $27 \mathrm{~mL}$ at its minimum. Predicted maximum and minimum volumes and their changes during active and passive emptying were all within 1 $\mathrm{SD}$ of the measured in vivo averages (Table 2), and

TABLE 2. Comparison of model-predicted measures of global function (volumes, changes in volume, fractional measures) in the normal left atrium to in vivo averages reported in Moyer et al. ${ }^{28}$.

\begin{tabular}{lccc}
\hline Metric & Simulation & Measured & Error (\%) \\
\hline$V_{\max }(\mathrm{mL})$ & 66 & $71 \pm 18$ & 7 \\
$V_{\min }(\mathrm{mL})$ & 27 & $29 \pm 11$ & 7 \\
$V_{\mathrm{preA}}(\mathrm{mL})$ & 39 & $44 \pm 12$ & 11 \\
$\Delta V_{\mathrm{t}}(\mathrm{mL})$ & 39 & $42 \pm 11$ & 7 \\
$\Delta V_{\mathrm{p}}(\mathrm{mL})$ & 27 & $27 \pm 8$ & $<1$ \\
$\Delta V_{\mathrm{a}}(\mathrm{mL})$ & 12 & $15 \pm 6$ & 20 \\
$\mathrm{EF}$ & $59(\%)$ & $60 \pm 9$ & 2 \\
$\mathrm{EF} F_{\mathrm{p}}(\%)$ & 31 & $38 \pm 7$ & 8 \\
$E F_{\mathrm{a}}(\%)$ & 31 & $34 \pm 12$ & 9 \\
$V_{\mathrm{p} / \mathrm{t}}(\%)$ & 31 & $65 \pm 10$ & 6 \\
$V_{\mathrm{a} / \mathrm{t}}(\%)$ & $35 \pm 10$ & 11 \\
\hline
\end{tabular}

$V_{\text {max }}$ : maximum volume; $V_{\min }$ : minimum volume; $V_{\text {preA }}$ : volume just before the onset of active emptying; $\Delta V_{\mathrm{t}}$ : total change in volume; $\Delta V_{\mathrm{p}}$ : change in volume during passive emptying; $\Delta V_{\mathrm{a}}$ : change in volume during active emptying; $\mathrm{EF}_{\mathrm{t}}$ : total emptying fraction; $\mathrm{EF}_{\mathrm{a}}$ : active emptying fraction; $\mathrm{EF}_{\mathrm{p}}$ : passive emptying fraction; $V_{\mathrm{p} / \mathrm{t}}$ : passive emptying as a fraction of total; $V_{\mathrm{a} / \mathrm{t}}$ : active emptying as a fraction of total. most were within $10 \%$. The simulated volume vs. time curve fell within $1 \mathrm{SD}$ of the measured in vivo averages at every point in the cardiac cycle, but underestimated the time spent in diastasis prior to atrial contraction (Fig. 5a). Simulated regional emptying fraction $\left(\mathrm{REF}_{\mathrm{t}}\right)$ was within $1 \mathrm{SD}$ of measured in vivo averages in four of five regions (Fig. 5b). The model produced a simulated left ventricular stroke volume of $70 \mathrm{~mL}$, end-diastolic volume of $121 \mathrm{~mL}$, and an ejection fraction of $58 \%$ consistent with reported normal values. ${ }^{24} \mathrm{MV}$ blood velocity (computed assuming a valve diameter of $2.75 \mathrm{~cm}$ ) was higher during passive emptying compared to active emptying (peaks of 58 vs. $39 \mathrm{~cm} / \mathrm{s}$ ), producing an E-to-A ratio of 1.5 , again matching reported values in normal subjects. ${ }^{4}$ As expected, $60 \%$ of ventricular filling occurred during early ventricular diastole (passive emptying of the atrium). PV blood
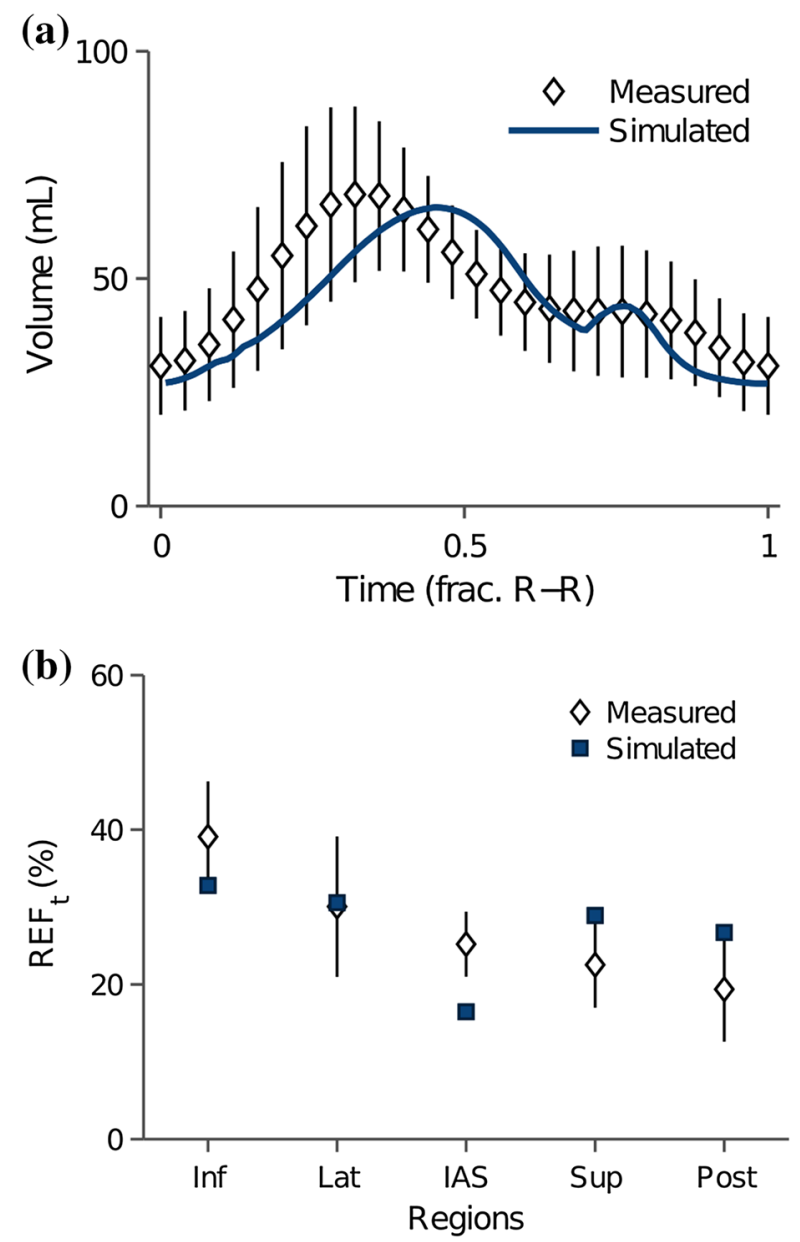

FIGURE 5. Validation of global and regional function in the normal left atrium model against measured in vivo averages from healthy subjects. (a) Global volume-time curves of baseline FE model (Simulated) compared to average curves from healthy subjects (Measured) (b) Regional emptying fraction $\left(R E F_{t}\right)$ of five anatomic regions in normal left atrial model compared to average in vivo measurements $(n=10$, mean \pm SD). 

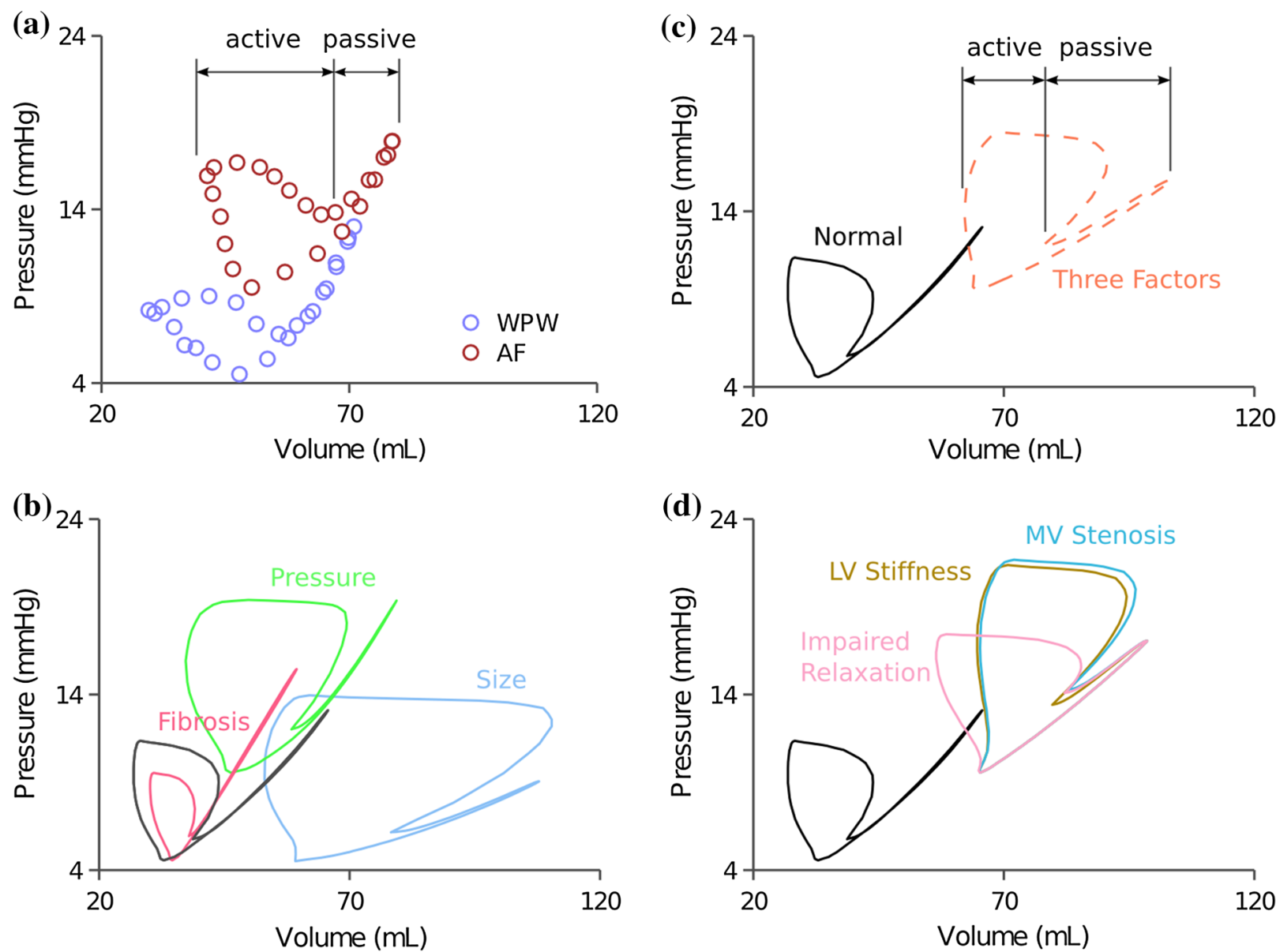

FIGURE 6. Model study of the effect of atrial and ventricular factors on the mechanics of paroxysmal AF. (a) Average pressurevolume loops from 23 AF patients compared to loops from 6 patients with Wolff-Parkinson-White syndrome, in whom atrial function is relatively normal. ${ }^{27} \mathrm{AF}$ loops are shifted to higher pressures with an increased reliance on active emptying. (b) Changes in model pressure-volume behavior due to higher pressure, larger size, and fibrosis compared to normal model (black); shape and conduction had little effect on atrial mechanics and are omitted for clarity. (c) The three-factor model (size, pressure, fibrosis) explained over $\mathbf{8 0} \%$ of the changes between healthy and AF hearts; note that volumes were validated against directly measured MRI volumes (Fig. 7c), which were larger than conductance volumes used to plot loops in panel (a). (d) Simulated impaired LV relaxation increased reliance on active emptying, while simulated mitral valve stenosis or increased passive LV stiffness increased pressures and reduced active emptying.

velocity (assuming a vein diameter of $0.8 \mathrm{~cm}$ ) during atrial filling $(58 \mathrm{~cm} / \mathrm{s})$ and passive emptying $(36 \mathrm{~cm} / \mathrm{s})$ matched Doppler studies. ${ }^{4}$

\section{Effect of Atrial Factors on Predicted AF Mechanics}

Changes in size and pressure had the largest influence on function (Fig. 6) while changes in atrial shape and electrical conduction had negligible effects. As expected, increasing atrial size shifted the $P-V$ loop rightward in the $P-V$ plane; a larger atrium was also more compliant, experiencing smaller pressure changes during passive filling and emptying and therefore accomplishing a smaller fraction of its emptying during the passive phase (Fig. 6b). Increasing atrial pressure shifted the $P-V$ loops upward and rightward on the $P-$ $V$ plane, reducing passive filling volume and increasing passive filling pressure as the atrium operated on a "steeper" portion of the passive curve (Fig. 6b). Simulated fibrosis had a smaller effect on atrial mechanics compared to size and pressure, but did alter both passive and active function. As expected, fibrosis created a steeper passive $P-V$ curve; somewhat surprisingly, fibrosis also reduced active stroke volume and active emptying as a fraction of total (Fig. 6b). Combining levels of dilation, increased pressure, and fibrosis typical of AF produced passive and active mechanics quite similar to our reported measurements in patients with paroxysmal AF, explaining $84 \%$ of the observed difference between healthy subjects and these patients in pressures and volumes at the maximum, minimum, and pre-contractile states (Fig. 6c; note that the conductance catheter volumes used to generate the AF PV loop in Fig. 6a were lower than those measured 

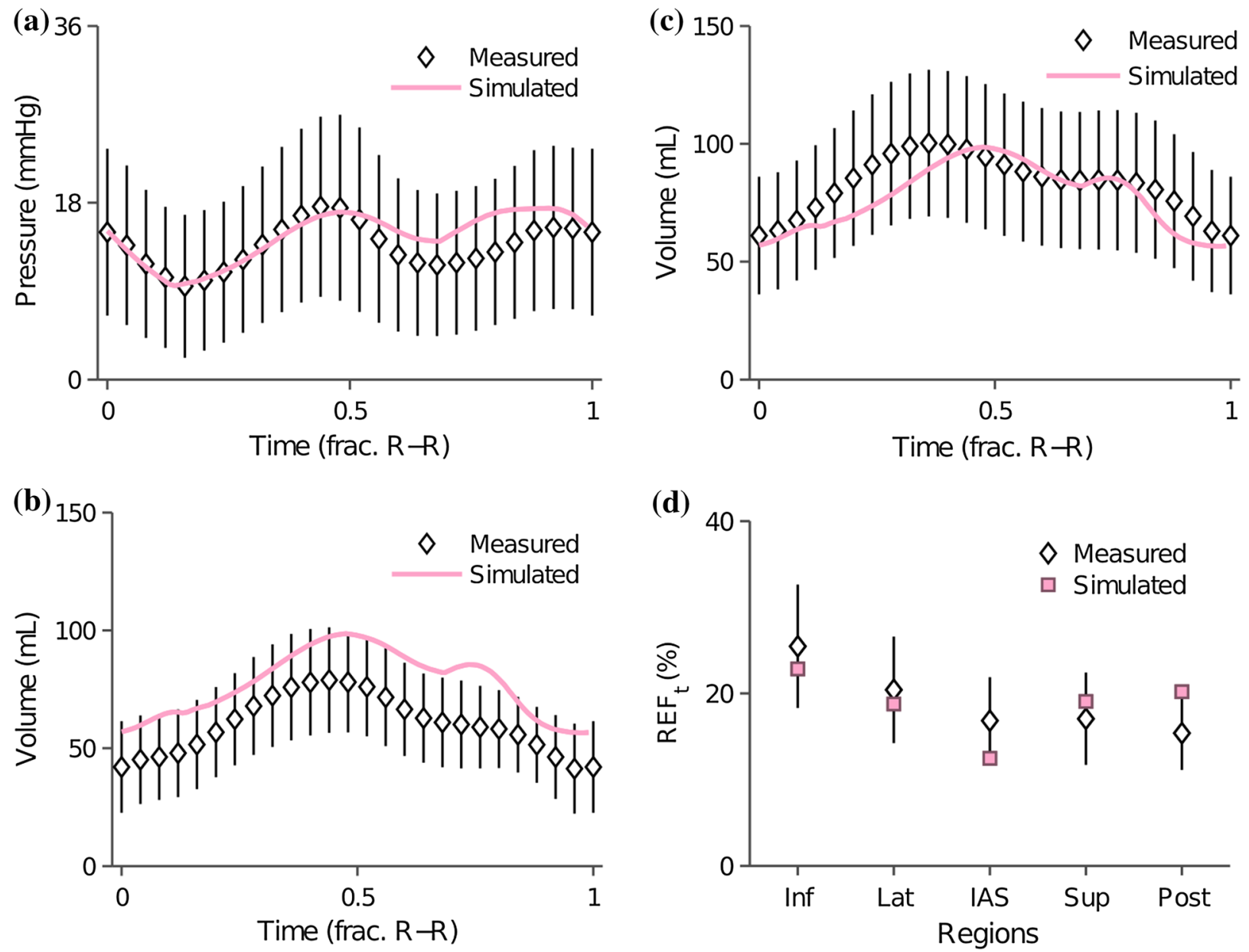

FIGURE 7. Comparison of AF model including all five atrial factors plus impaired relaxation to measurements from patients with paroxysmal AF. (a) Pressure-time curves agreed well with invasive measurements in 23 patients immediately prior to ablation. (b) Conductance-catheter-derived volumes in same patients (Measured) were smaller than those measured directly by MRI and shown in panel (c); MRI-derived volumes were used for validation as explained in text. (c) Volume-time curves fell within one standard deviation of the average measured from MRI in 31 patients (Measured, mean \pm SD) throughout the cardiac cycle. (d) Regional emptying fraction $\left(R E F_{t}\right.$ ) of five anatomic regions in the final AF model fell within 1 SD of the in vivo averages derived from MRI in 31 AF patients.

directly by MRI and used for validation, see below and Fig. 7).

\section{Effect of Ventricular Factors on Predicted AF Mechanics}

Although the model incorporating the most important atrial factors (dilation + pressure + fibrosis) explained most of the altered function we observed in patients with paroxysmal $\mathrm{AF}$, it missed one striking feature: a dramatic increase in the fraction of atrial emptying that occurs during the active phase in AF patients compared to healthy subjects (Fig. 6). We therefore considered whether left ventricular factors that might disrupt passive emptying of the atrium could reproduce this increased reliance on active emptying in our coupled model (Fig. 6d). Beginning with a model including all 5 atrial factors discussed above, we modeled an increase in passive LV stiffness by doubling the exponential coefficient in the passive elastance curve in the circuit model, which increased $\mathrm{LV}$-end diastolic pressure from 8 to $22 \mathrm{mmHg}$. We modeled mitral valve stenosis by quadrupling the resistance across the mitral valve in the hydraulic circuit, which reduced peak mitral valve flow by over $60 \%$ and the $E: A$ ratio from 1.47 to 0.88 . Finally, we modeled delayed LV relaxation as in Hay et al. ${ }^{16}$ by extending the active elastance curve to include an exponential decay to zero with a time constant tau $=80 \mathrm{~ms}$ at the beginning of diastole. We found that increased passive LV stiffness and increased resistance to flow through the mitral valve both impaired active atrial emptying more than passive emptying, while delayed LV relaxation selectively affected 
atrial passive emptying, increasing the relative importance of active emptying as observed in patients with paroxysmal AF (Fig. 6d). Delayed LV relaxation resulted in total emptying fraction of $43 \%$ and an active portion of $61 \%$, both within $1 \%$ of measured averages. The final AF model incorporating five atrial factors plus impaired LV relaxation reproduced measured invasive pressure-time curves (Fig. 7a), MRI volume-time curves (Fig. 7c), and regional EF in all regions of the atrium (Fig. 7d) within one standard deviation. Comparison of Figs. $7 \mathrm{~b}$ and $7 \mathrm{c}$ reveals that conductance catheter volumes were lower and followed a slightly different time course compared to volumes measured directly by MRI; as noted under Methods, we gave preference to directly measured MRI volumes when validating the model because conductance volumes were calibrated against pre-procedure MRI performed on a different day.

\section{DISCUSSION}

Atrial fibrillation (AF) is a rhythm disorder with rapidly increasing prevalence due to the aging of the population. In addition to an increased risk of stroke, AF triggers structural remodeling of the atrium and a gradual loss of atrial function. However, the relative contributions of specific features of AF-induced remodeling to changes in atrial mechanical function are unclear. We therefore constructed and validated a finite-element model (FEM) of the normal human left atrium and coupled the FEM to a hydraulic circuit model to capture the hemodynamic interactions between the atrium, pulmonary circulation, and left ventricle. The normal model reproduced measured volumes within $1 \mathrm{SD}$, as well as most metrics of regional mechanics. Using this validated human model as a starting point, we explored the impact of individual features of atrial remodeling on atrial mechanics and found that a combination of dilation, increased pressure, and fibrosis can explain most of the observed changes in mechanics in patients with paroxysmal AF. However, only impaired ventricular relaxation could reproduce in the model the increased reliance on active emptying we observed in these patients.

\section{Determinants of Atrial Mechanics in Normal Subjects and Paroxysmal AF Patients}

Given the fact that both volumes and pressures roughly double in AF compared to healthy subjects, ${ }^{20,35}$ it was not surprising that dilation and pressure had the largest effects on predicted atrial mechanics in our model. However, several other model findings were more surprising. First, in the early stages of developing the model we found that the boundary conditions imposed by surrounding structures were much more important in determining atrial shape and wall motion than we had expected. We found we needed to represent not only the attachments to the pulmonary veins and the ventricular forces acting on the mitral annulus, but also septal forces due to right atrial pressure and contact with the aortic root and chest wall in order to match both global and regional volume changes through the cardiac cycle (Fig. 5).

When exploring the basis for changes in mechanics with atrial fibrillation, we were surprised that introducing the more spherical shape of AF atria had little effect on predicted mechanics; however, the effects of shape on wall stress distributions proved to be much smaller than the effects of pressure and dilation. Another surprise was that when we incorporated fibrosis into our model in order to better match measured passive filling and emptying (Fig. 6), fibrosis also caused decreased active emptying. This appears to be due to the Frank-Starling mechanism: by reducing passive filling and strain at the start of contraction, fibrosis reduced length-dependent active tension generation. One interesting implication of this finding is that anti-fibrotic therapy in AF patients might paradoxically increase dependence on active emptying.

Finally, we were quite surprised to find that none of the atrial factors we considered-alone or in combination - could explain the dramatically increased reliance on active emptying we observed in patients with paroxysmal AF $(61 \%$ of total emptying vs. $32 \%$ in healthy subjects). In fact, the only atrial or ventricular factor we tested that could reproduce this behavior in the model was impaired LV relaxation. The results of our simulations of LV factors are consistent with some prior reports that quantified atrial function in patients with left ventricular diseases but no AF. For instance, impaired ventricular relaxation caused by ischemia, prior MI, and angina increased the contribution of active atrial emptying to LV stroke volume from 26 to $38 \%$, while restricted ventricular filling in cardiomyopathy patients reduced the contribution from 26 to $18 \%$, as the atrium pumped against larger afterloads during its active phase. ${ }^{31}$

\section{Coupling Between the Left Atrium, Left Ventricle, and Pulmonary Circulation}

Early in our consideration of changes associated with AF-induced remodeling, we were forced to consider how to represent coupling between the atrium, the left ventricle, and the pulmonary circulation. For example, when simulating the increase in pressures associated with AF, we initially maintained the same 
displacement boundary conditions at the mitral valve as for the normal atrium. Yet maintaining those displacements required a much larger force, even though it seems highly unlikely that the ventricles of AF patients generate much higher forces. We therefore chose to represent physical LV-atrial coupling across the mitral valve by assuming constant forces applied by the LV to the various atrial models rather than prescribing displacements. Similarly, when simulating the severe dilation of the atrium associated with AF, it became immediately apparent that simply loading each model to the same pressures was an unacceptable approach: because the volume of the dilated atrium increased much more for a given change in pressure, passive filling and emptying volumes at matched pressures far exceeded plausible stroke volumes. Therefore, we opted to couple the FEM to a hydraulic circuit to simulate the actual pressures that would be expected at each phase of atrial filling given not only the properties of the atrium but also upstream (pulmonary veins) and downstream (left ventricle) hemodynamic coupling.

We chose to couple the models by passing enddiastolic and end-systolic pressure relationships from the FEM to the circuit model, and pressures from the circuit model to the FEM, rather than dynamically updating pressures throughout the FE simulation using the circuit model, as proposed for the left ventricle by Kerckhoffs. ${ }^{23}$ The major advantage of our approach is computational efficiency - we estimate that dynamic coupling would have increased run times by roughly a factor of 100 . Because history-dependent effects such as passive viscoelasticity and shortening de-activation likely play relatively minor roles in the mechanics of AF compared to the large changes in loading, size, and material properties considered here, we felt the improved accuracy from dynamic coupling would not be worth the computational cost.

\section{Comparison to Previous Models of Atrial Mechanics}

To our knowledge, this is the first finite-element model of human left atrial mechanics validated in both normal subjects and patients with a history of atrial fibrillation that takes into account coupling to the pulmonary circulation and left ventricle. However, Hunter et al. did construct a finite-element model of the human atrium to explore correlations between wall stress and electrophysiological changes. ${ }^{19}$ They computed von Mises stresses of 26-52 $\mathrm{kPa}$ in the atrial wall at a pressure of $20 \mathrm{mmHg}$, which compares well to von Mises stresses of $8-27 \mathrm{kPa}$ in our model at a $2.5 \times$ lower filling pressure. We also identified some of the same locations of peak wall stresses reported by Hunter, including in the septal wall and near the pulmonary vein ostia. Other published models include a model of interactions between the atrium and interventional devices ${ }^{22}$ and a comprehensive finite-element model of the porcine atria based on computed tomography imaging by Di Martino and colleagues. ${ }^{6,7}$ Average principal stresses in that model were $35 \%$ lower than our average of $17 \mathrm{kPa}$; this discrepancy likely stemmed from differences in geometry (the porcine model had a large appendage and did not include atrial fibers) and loading (the porcine model had a maximum pressure of $10 \mathrm{mmHg}$ ).

\section{Limitations and Future Work}

There are several limitations of the model presented here that should be noted. First, the normal atrial model was loaded with average pressures from the literature rather than pressures measured simultaneously with geometry and wall motion, owing to the invasive nature of atrial pressure measurement. The fact that regional ejection fraction in the septum of the model was outside the range of the experimental data may be due to this limitation, since the pressures acting on the septum were estimated. This conjecture is further supported by the fact that we were able to match regional motion in all regions in $\mathrm{AF}$ patients, in whom we were able to obtain both MRI and pressure data. A second limitation is that the model does not accurately reproduce diastasis, the pause in flow from the LA to $\mathrm{LV}$ between the passive and active phases of atrial emptying; instead, model volumes reach a brief minimum and then rise again as the atrium begins to generate pressure (Figs. 5, 6, and 7). Shortening the duration of active force generation by $25 \%$ and prolonging passive emptying exacerbated this artifact rather than remedying it, suggesting that the constant upstream pressure in the pulmonary veins may be the primary driver of this model behavior. Another limitation is the use of a simplified fiber structure. Zhao and coworkers have recently incorporated their detailed atrial fiber orientation data directly into electrophysiologic models, ${ }^{38}$ while other groups have utilized rule-based algorithms to generate detailed atrial fiber structures $;{ }^{8}$ incorporating similarly detailed fiber geometries into our FEM could potentially improve the accuracy of predictions in some regions. More broadly, the model presented here was constructed and validated using data from paroxysmal $\mathrm{AF}$, a relatively mild form of the disease characterized by intermittent bouts of fibrillation; we chose to focus on these patients because it was possible to obtain MRI and hemodynamic data during periods when the atrium was not actively fibrillating. However, the full clinical spectrum of AF includes such a wide range of 
degrees of remodeling, fibrosis, etc. that no one model can hope to represent the mechanics of all AF patients.

\section{CONCLUSIONS}

In order to better understand the role of remodeling induced by atrial fibrillation on mechanical function of the atrium, we constructed and validated a finite-element model of the human left atrium and used it to explore the impact of changes in atrial size, shape, pressures, fibrosis, and conduction velocity. We coupled our finite-element model to a circulation model to account for changes in filling and emptying due to both atrial and systemic factors. We were able to reproduce global and regional volume changes in normal subjects and patients with paroxysmal AF. Furthermore, model simulations suggested that in these patients (1) dilation and increased pressures in the atrium are likely the most important atrial factors in reducing atrial function; and (2) the increased reliance on active emptying appears to be due to impaired ventricular relaxation rather than changes in the atrium itself. We hope these findings will improve understanding of the mechanics of $\mathrm{AF}$ and guide development of future therapies.

\section{ACKNOWLEDGMENTS}

The authors wish to thank Dr. David Lopez for processing atrial MRI and pressure-volume loops. This work was supported in part by a Pre-Doctoral Fellowship (Christian Moyer) and an Established Investigator Award (Jeffrey Holmes) from the AHA, and NIH/NHLBI R01 HL-085160 (Jeffrey Holmes).

\section{REFERENCES}

\footnotetext{
${ }^{1}$ Alexander, J., K. Sunagawa, N. Chang, and K. Sagawa. Instantaneous pressure-volume relation of the ejecting canine left atrium. Circ. Res. 61:209-219, 1987.

${ }^{2}$ Bellini, C., and E. S. Di Martino. A mechanical characterization of the porcine atria at the healthy stage and after ventricular tachypacing. J. Biomech. Eng. 134:021008, 2012.

${ }^{3}$ Blume, G. G., C. J. Mcleod, M. E. Barnes, J. B. Seward, P. A. Pellikka, P. M. Bastiansen, and T. S. M. Tsang. Left atrial function: physiology, assessment, and clinical implications. Eur. J. Echocardiogr. 12:421-430, 2011.

${ }^{4}$ Boyd, A. C., N. B. Schiller, D. Leung, D. L. Ross, and L. Thomas. Atrial dilation and altered function are mediated by age and diastolic function but not before the eighth decade. JACC Cardiovasc. Imaging 4:234-242, 2011.

${ }^{5}$ Clark, D. M., V. J. Plumb, A. E. Epstein, and G. N. Kay. Hemodynamic effects of an irregular sequence of ventricular
}

cycle lengths during atrial fibrillation. J. Am. Coll. Cardiol. 30:1039-1045, 1997.

${ }^{6}$ Di Martino, E. S., C. Bellini, and D. S. Schwartzman. In vivo porcine left atrial wall stress: computational model. $J$. Biomech. 44:2589-2594, 2011.

${ }^{7}$ Di Martino, E. S., C. Bellini, and D. S. Schwartzman. In vivo porcine left atrial wall stress: effect of ventricular tachypacing on spatial and temporal stress distribution. $J$. Biomech. 44:2755-2760, 2011.

${ }^{8}$ Doessel, O., M. W. Krueger, F. M. Weber, M. Wilhelms, and G. Seemann. Computational modeling of the human left atrial anatomy and electrophysiology. Med. Biol. Eng. Comput. 50:773-799, 2012.

${ }^{9}$ Fomovsky, G. M., and J. W. Holmes. Evolution of scar structure, mechanics, and ventricular function after myocardial infarction in the rat. Am. J. Physiol. Heart Circ. Physiol. 298:H221-H228, 2010.

${ }^{10}$ Frost, L. Lone atrial fibrillation: good, bad, or ugly? Circulation 115:3040-3041, 2007.

${ }^{11}$ Gloschat, C., J. Cates, B. Walker, and R. S. MacLeod. Statistical shape modeling of the left atrium from MRI of patients with atrial fibrillation. J. Cardiovasc. Magn. Reson. 13:P57, 2011.

${ }^{12}$ Go, A. S., E. M. Hylek, K. A. Phillips, Y. Chang, L. E. Henault, J. V. Selby, and D. E. Singer. Prevalence of diagnosed atrial fibrillation in adults: national implications for rhythm management and stroke prevention: the anticoagulation and risk factors in atrial fibrillation (ATRIA) study. JAMA 285:2370-2375, 2001.

${ }^{13}$ Govindjee, S., and P. A. Mihalic. Computational methods for inverse finite elastostatics. Comput. Methods Appl. Mech. Eng. 136:47-57, 1996.

${ }^{14}$ Guccione, J. M., and A. D. McCulloch. Mechanics of active contraction in cardiac muscle: part I-constitutive relations for fiber stress that describe deactivation. J. Biomech. Eng. 115:72-81, 1993.

${ }^{15}$ Guyton, A. C., A. W. Lindsey, B. Abernathy, and T. Richardson. Venous return at various right atrial pressures and the normal venous return curve. Am. J. Physiol. 189:609-615, 1957.

${ }^{16}$ Hay, I., J. Rich, P. Ferber, D. Burkhoff, and M. S. Maurer. Role of impaired myocardial relaxation in the production of elevated left ventricular filling pressure. Am. J. Physiol. Heart Circ. Physiol. 288:H1203-H1208, 2005.

${ }^{17}$ Ho, S. Y., R. H. Anderson, and D. Sánchez-Quintana. Atrial structure and fibres: morphologic bases of atrial conduction. Cardiovasc. Res. 54:325-336, 2002.

${ }^{18}$ Ho, S. Y., D. Sanchez-Quintana, J. A. Cabrera, and R. H. Anderson. Anatomy of the left atrium: implications for radiofrequency ablation of atrial fibrillation. J. Cardiovasc. Electrophysiol. 10:1525-1533, 1999.

${ }^{19}$ Hunter, R. J., Y. Liu, Y. Lu, W. Wang, and R. J. Schilling. Left atrial wall stress distribution and its relationship to electrophysiologic remodeling in persistent atrial fibrillation. Circ. Arrhythm. Electrophysiol. 5:351-360, 2012.

${ }^{20}$ Jahnke, C., J. Fischer, J.-H. Gerds-Li, R. Gebker, R. Manka, E. Fleck, I. Paetsch, and C. Kriatselis. Serial monitoring of reverse left-atrial remodeling after pulmonary vein isolation in patients with atrial fibrillation: a magnetic resonance imaging study. Int. J. Cardiol. 153:42-46, 2011.

${ }^{21}$ Jaïs, P., J. T. Peng, D. C. Shah, S. Garrfgue, M. Hocini, T. Yamane, M. Haissaguerre, S. S. Barold, R. Roudaut, and J. Clementy. Left ventricular diastolic dysfunction in patients with so-called lone atrial fibrillation. J. Cardiovasc. Electrophysiol. 11:623-625, 2000. 
${ }^{22}$ Jernigan, S. R., G. D. Buckner, J. W. Eischen, and D. R. Cormier. Finite element modeling of the left atrium to facilitate the design of an endoscopic atrial retractor. $J$. Biomech. Eng. 129:825-837, 2007.

${ }^{23}$ Kerckhoffs, R. C. P., M. L. Neal, Q. Gu, J. B. Bassingthwaighte, J. H. Omens, and A. D. McCulloch. Coupling of a 3D finite element model of cardiac ventricular mechanics to lumped systems models of the systemic and pulmonic circulation. Ann. Biomed. Eng. 35:1-18, 2007.

${ }^{24}$ Lorenz, C. H., E. S. Walker, V. L. Morgan, S. S. Klein, and T. P. Graham. Normal human right and left ventricular mass, systolic function, and gender differences by cine magnetic resonance imaging. J. Cardiovasc. Magn. Reson. 1:7-21, 1999.

${ }^{25}$ Maas, S. A., B. J. Ellis, G. A. Ateshian, and J. A. Weiss. FEBio: finite elements for biomechanics. J. Biomech. Eng. 134:011005, 2012.

${ }^{26}$ Markides, V., R. J. Schilling, S. Yen Ho, A. W. C. Chow, D. W. Davies, and N. S. Peters. Characterization of left atrial activation in the intact human heart. Circulation 107:733-739, 2003.

${ }^{27}$ Moyer, C. B. Mechanical function of the left atrium. PhD Thesis, University of Virginia, 2013. http://libra.virginia.edu/catalog/libra-oa:3938.

${ }^{28}$ Moyer, C. B., P. Helm, C. J. Clarke, L. P. Budge, C. M. Kramer, J. D. Ferguson, P. T. Norton, and J. W. Holmes. Wall-motion based analysis of global and regional left atrial mechanics. IEEE Trans. Med. Imaging 32:1765-1776, 2013.

${ }^{29}$ Nattel, S. New ideas about atrial fibrillation 50 years on. Nature 415:219-226, 2002.

${ }^{30}$ Platonov, P. G., L. B. Mitrofanova, V. Orshanskaya, and S. Y. Ho. Structural abnormalities in atrial walls are associated with presence and persistency of atrial fibrillation but not with age. J. Am. Coll. Cardiol. 58:2225-2232, 2011.
${ }^{31}$ Prioli, A., P. Marino, L. Lanzoni, and P. Zardini. Increasing degrees of left ventricular filling impairment modulate left atrial function in humans. Am. J. Cardiol. 82:756-761, 1998.

${ }^{32}$ Raghavan, M. L., B. Ma, and M. F. Fillinger. Non-invasive determination of zero-pressure geometry of arterial aneurysms. Ann. Biomed. Eng. 34:1414-1419, 2006.

${ }^{33}$ Sanfilippo, A., V. Abascal, M. Sheehan, L. Oertel, P. Harrigan, R. Hughes, and A. Weyman. Atrial enlargement as a consequence of atrial fibrillation: a prospective echocardiographic study. Circulation 82:792-797, 1990.

${ }^{34}$ Schwartzman, D., J. Lacomis, and W. G. Wigginton. Characterization of left atrium and distal pulmonary vein morphology using multidimensional computed tomography. J. Am. Coll. Cardiol. 41:1349-1357, 2003.

${ }^{35}$ Stefanadis, C., J. Dernellis, C. Stratos, E. Tsiamis, C. Tsioufis, K. Toutouzas, C. Vlachopoulos, C. Pitsavos, and P. Toutouzas. Assessment of left atrial pressure-area relation in humans by means of retrograde left atrial catheterization and echocardiographic automatic boundary detection: effects of dobutamine. J. Am. Coll. Cardiol. 31:426-436, 1998.

${ }^{36}$ Stefanadis, C., J. Dernellis, C. Stratos, E. Tsiamis, C. Vlachopoulos, K. Toutouzas, S. Lambrou, C. Pitsavos, and P. Toutouzas. Effects of balloon mitral valvuloplasty on left atrial function in mitral stenosis as assessed by pressure-area relation. J. Am. Coll. Cardiol. 32:159-168, 1998.

${ }^{37}$ Wolf, P., R. Abbott, and W. Kannel. Atrial fibrillation as an independent risk factor for stroke: the Framingham study. Stroke 22:983-988, 1991.

${ }^{38}$ Zhao, J., T. D. Butters, H. Zhang, I. LeGrice, G. B. Sands, and B. H. Smaill. Image-based model of atrial anatomy and electrical activation: a computational platform for investigating atrial arrhythmia. IEEE Trans. Med. Imaging 32:1827, 2013. 